\title{
28 Research Square \\ Escherichia coli harboring mcr-1 on a Novel Plasmid in a Mink
}

\author{
Weishan Chang \\ Shandong Agricultural University \\ Xinxing Wang \\ Shandong Agricultural University \\ Jun Peng ( $\sim$ jpeng@sdau.edu.cn ) \\ Shandong Agricultural University
}

\section{Research Article}

Keywords: mcr-1, mink, Plasmid, Escherichia coli

Posted Date: March 4th, 2021

DOI: https://doi.org/10.21203/rs.3.rs-266792/v1

License: (1) This work is licensed under a Creative Commons Attribution 4.0 International License. Read Full License 


\section{Abstract}

Background: Antimicrobial resistant Escherichia coli from mink can be transmitted to human through water environment. Therefore, it is necessary to routinely detect the presence of antimicrobial resistance genes of mink.

Methods: In the current study, we described the characterization of an mcr-1-positive Escherichia coli isolate from a dead mink in Dongping Lake, China. The molecular characterization of plasmids was also investigated.

Results: The strain was resistant to colistin, but it remained susceptible to several other agents, including amikacin, piperacillin-tazobactam and all carbapenems. Escherichia coli Mink_1 belonged to sequence type 140. Whole genome sequencing(WGS) showed that two plasmid, pM1 mcr and pM1ctx, coexisted in the same bacterial. Blast and phylogenetic tree imply that epidemic plasmids are vehicles for the dissemination of colistin resistance genes among the Enterobacterale.

Conclusion: Restrictive and rational use of antibiotics in animal husbandry should be taken to reduce the dissemination of mcr-1.

\section{Introduction}

The discovery of a colistin resistance gene, $m c r-1$, in China may herald the emergence of pandrugresistant bacteria(Liu et al., 2016). The gene has been found primarily in Escherichia coli (E. coli) but has also been identified in other members of the Enterobacterales, in human, animal, food, and environmental samples in over 50 countries on every continent except Antarctica (Fernandes, M., 2016; Rapoport, M., 2016; Skov, R., 2016; Zeng, K., 2016). However, the prevalence and characteristics of E.coli from mink was rarely reported. Here, we report the presence of $m c r-1$ in an $E$. coli strain cultured from a mink in Dongping Lake, China.

\section{Methods}

E. coli Mink_1 was cultured from a dead mink which presented to a veterinary station in Dongping, China on 12th, July 2019. The strain of E. coli was isolated from liver, where bleeding spots were found. Since our research did not hurt living animals, ethical approval was not required for the study.

The isolate was forwarded to SDAU for susceptibility testing. Antimicrobial susceptibility testing (AST) of the isolate was performed using broth dilution method. The minimum inhibitory concentrations (MICs) of colistin for the $E$. coli isolate was also tested by the broth dilution method. The result interpretations were based on Clinical and Laboratory Standards Institute guidelines (CLSI,2021), except for colistin, where European Committee on Antimicrobial Susceptibility Testing guidelines breakpoints $(\geq 2 \mu \mathrm{g} / \mathrm{mL})$ were used(EUCAST, 2021). 
Whole genome sequencing(WGS) of E. coli Mink_1 was performed using a Nanopore technology and a sequence platform GridION. Complete Genome Sequencing was used to determine the location of the resistance genes. When we obtained the genome or plasmid sequences, we compared the sequences with resistance gene sequences. If the Alignment showed above $95 \%$ identity, we speculated that the resistance genes located on the responding sequence. The bioinformatics tools included Ediseq, Blastn, and Snapgene. By Blastn, we analysis the identity and coverage of our plasmid with pHNSHP45.

The replicon types of plasmids were determined by PCR-based replicon typing (PBRT)(Carattoli, A,2005). The bioinformatics tools uesd include Megalign, Blastn, plasmid finder, Snapgene, etc.

The sequences of $m c r-1$-postive plasmids was downloaded from GenBank. Then the similar sequence were cut for comparison. The construction of the phylogenetic tree was performed by the maximum likelihood method using MegAlign 8.1.4 (DNAstar Co., Ltd).

\section{Results}

E. coli Mink_1 expressed a level of high resistance to seven different antimicrobial compounds in addition to colistin (MIC = $8 \mathrm{mg} / \mathrm{L})$ (Table 1$)$. To note, it has a MIC of Sulfanilamide of $>4 \mu \mathrm{g} / \mathrm{mL}$. 
Table 1 Antibiotic resistance profile of E. coli Mink_1

\begin{tabular}{ll}
\hline Antibiotic(s) & $\mathrm{MIC}(\mathrm{s})(\mu \mathrm{g} / \mathrm{ml})$ \\
\hline Amikacin & $8, \mathrm{~S}$ \\
Amoxicillin/clavulanate & $16 / 8, \mathrm{I}$ \\
Ampicillin & $>16, \mathrm{R}$ \\
Aztreonam & $>16, \mathrm{R}$ \\
Cefazolin & $>16, \mathrm{R}$ \\
Cefepime & $>16, \mathrm{R}$ \\
Ceftazidime & $>16, \mathrm{R}$ \\
Ceftriaxone & $>32, \mathrm{R}$ \\
Ciprofloxacin & $>2, \mathrm{R}$ \\
Colistin & $8, \mathrm{R}$ \\
Ertapenem & $0.25, \mathrm{~S}$ \\
Gentamicin & $>8, \mathrm{R}$ \\
Imipenem & $0.25, \mathrm{~S}$ \\
Levofloxacin & $>4, \mathrm{R}$ \\
Meropenem & $0.25, \mathrm{~S}$ \\
Nitrofurantoin & $16, \mathrm{~S}$ \\
Piperacillin-tazobactam & $4 / 4, \mathrm{~S}$ \\
Tetracycline & $>8, \mathrm{R}$ \\
Tobramycin & $>8, \mathrm{R}$ \\
Trimethoprim-sulfamethoxazole & $>2 / 38, \mathrm{R}$ \\
Sulfanilamide & \\
\hline & \\
\hline &
\end{tabular}

$\mathrm{R}=$ resistant $\mathrm{I}=$ intermediate, and $\mathrm{S}=$ susceptible.

E. coli Mink_1 belonged to sequence type 140 (ST140), a rare E. coliST. The two plasmid, pM1mcr and pM1ctx, coexisted in the same bacterial. pM1 mcr, was $238 \mathrm{~kb}$ in size and BLAST analysis and referring to GenBank indicated that pM1 mcr was identified and found to be of an IncF type that encoded a mcr-1 gene along with six additional antimicrobial resistance genes, including the ESBL gene $b / a_{\mathrm{CTX}-\mathrm{M}-14}$, and tetA, SUl2, b/a $a_{\mathrm{TEM}}$. The main reploins we found was FIA and FIB.

Results of phylogenetic tree of pM1mcr construction was shown in Fig.1. MH522419, the most similar sequence to pM1mcr, was a strain of mcr-1-harboring Salmonella from diarrhoeal outpatients in Shanghai, China.

The second plasmid, pM1ctx,was $116 \mathrm{~kb}$ in size and was assigned to IncN, which carried antibiotic resistance genes $a p h\left(3^{\prime}\right)-l a$ and bla CTX-M-7 $_{\text {(Fig.2). }}$. 
Blastn analysis showed that pM1mcr had a query coverage of $94 \%$ and maximal $99 \%$ identity to pHNSHP45, the first plasmid reported to harbor mcr-1.

Nucleotide sequence accession numbers. The whole genome sequence data of the mcr-7-positive Escherichia coli have been deposited in the Genome Warehouse in National Genomics Data Center, Beijing Institute of Genomics (BIG), Chinese Academy of Sciences, under accession number GWHACBG00000000 that is publicly accessible at https://bigd.big.ac.cn/gwh. The whole genome sequence data of the plasmid of mcr-1-positive E.coli have been deposited in the Genome Warehouse in National Genomics Data Center, Beijing Institute of Genomics (China National Center for Bioinformation), Chinese Academy of Sciences, under accession number GWHASJA00000000 that is publicly accessible at https://bigd.big.ac.cn/gwh.

\section{Discussion}

According to the present data from GenBank, the mcr-1-positive plasmids were mainly from China, with a percentage of $52.3 \%$, and $E$. coli is the primary host of $m c r-1$ in China. mcr-1 mainly exists in livestock breeding and animal derived food in China which varied from $1.5 \sim 30.9 \%$ in different years (Li, 2018). He Jie detected 611 strains of Escherichia coli from 23 hospitals in 11 cities in China, and the prevalence of mcr-1 in hospital was $0.98 \sim 1.5 \%$ in hospital, which is significantly lower than that in animal husbandry, but it is worthy of people's attention because it shows that drug-resistant genes are prevalent and spread among people(He. 2018).

The E.coli was isolated from liver of a mink. Before the death of the mink, it showed depression and liver bleeding spots were found after autopsy. So we speculated that the strain was pathogen, and further test should be done to determine whether the strain is pathogenic or not. The lethal pathogen may be EPEC from the environment. Meanwhile, it may also be conditional pathogen in vivo.

The association between mcr-1 and epidemic plasmids is concerning, as epidemic plasmids like IncF are vehicles for the dissemination of colisitn resistance genes among the Enterobacterales.

\section{Conclusion}

Continued surveillance to determine the true frequency for $m c r-1$ is critical and measures including restrictive use of antibiotics in animal husbandry should be taken to reduce the dissemination of $\mathrm{mcr}-1$. Restrictive and rational use of antibiotics in animal husbandry should be taken to reduce the dissemination of $m c r-1$.

\section{Declarations}

Ethics approval consent to participate 
E. coli Mink_1 was cultured from a dead mink which presented to a veterinary station. The mink was sent to high pressure sterilization after experiment. Since our research did not hurt living animals, ethical approval was not required for the study.

\section{Consent for publication}

Not applicable

\section{Availability of data and materials}

The datasets generated and/or analysed during the current study are available in the Genome Warehouse in National Genomics Data Center, Beijing Institute of Genomics, at https://bigd.big.ac.cn/gwh.

\section{Competing interests}

The authors declare that they have no competing interests

\section{Funding}

We acknowledge supports from National Natural Science Foundation of China (31672587), Key Research and Development Project of Shandong Province, China (2016GNC110013), Shandong Province Modern Agricultural Industrial Technology System Pig Innovation Team Project (SDAIT-08-09), and Program of School of Public Health, Shandong First Medical University \& Shandong Academy of Medical Sciences (201719)

\section{Authors' contributions}

XW wrote the main manuscript text. WC supervised the research. JP revised the manuscript and funded the experiments. All authors reviewed the manuscript.

\section{Acknowledgements}

Not applicable

\section{References}

Carattoli, A., Bertini, A., Villa, L., Falbo, V., Hopkins, K., \& Threlfall, EJ. (2005). Identification of plasmids by pcr-based replicon typing. Journal of Microbiological Methods, 63(3), 219-228.

CLSI (2021). Performance Standards for Antimicrobial Susceptibility Testing: Thirty-one Informational Supplement M100-S31. Wayne, PA. Clinical and Laboratory Standards Institute.

EUCAST (2021). Breakpoint Tables for Interpretation of MICs and Zone Diameters: Version 11.0. European Committee on Antimicrobial Susceptibility Testing. 
Fernandes, M., Moura, Q., Sartori, L., Silva, K., Cunha, M., Esposito, F., ... Milena Dropa. (2016). Silent dissemination of colistin-resistant Escherichia coli in South America could contribute to the global spread of the mcr-1 gene. Euro surveillance. 21(17).

He Jie. (2018). Prevalence of colistin-resistance gene mcr-1 in Enterobacterales from blood stream infections in Zhejiang Province.(Doctoral dissertation).

Li, Zhen., Jiang, Wen., Song, Chuan., Zhao Jian., Li, Yue., Chi L., ... Qu, Zhi. (2018). Colistin resistance status of escherichia coli and salmonella from swine and poultry and detection of $m c r-1$ gene. Progress in Veterinary Medicine.039(012), 20-26.

Liu, Y., Wang, Y., Walsh, R., Yi, L., Zhang, R., Spencer, J., ... Shen, J. (2016). Emergence of plasmidmediated colistin resistance mechanism mcr-1 in animals and human beings in China: a microbiological and molecular biological study. Lancet Infectious Disease. 16, pp. 161-168.

Rapoport, M. , Faccone, D. , Pasteran, F. , Ceriana, P. , Albornoz, E. , Petroni, A., \& Corso, A. (2016). mcr-1mediated colistin resistance in human infections caused by Escherichia coli: first description in Latin America. Antimicrobial Agents \& Chemotherapy, 60(7), pp. 4412-3.

Skov, R., \& Monnet, D. (2016). Plasmid-mediated colistin resistance ( $m c r-1$ gene): three months later, the story unfolds. Euro Survll, 21(9), 30155.

Thomson, K., \& Sanders, C. Detection of extended-spectrum beta-lactamases in members of the family Enterobacteriaceae: comparison of the double-disk and three-dimensional tests. Antimicrob Agents Chemother. 1992 Sep;36(9):1877-82.

Zeng, K., Doi, Y., Patil, S., Huang, X., \& Tian, G. (2016). Emergence of plasmid-mediated $m c r-1$ gene in colistin-resistant Enterobacter aerogenes and Enterobacter cloacae. Antimicrobial Agents and Chemotherapy, 60(6), 3862-3.

\section{Figures}

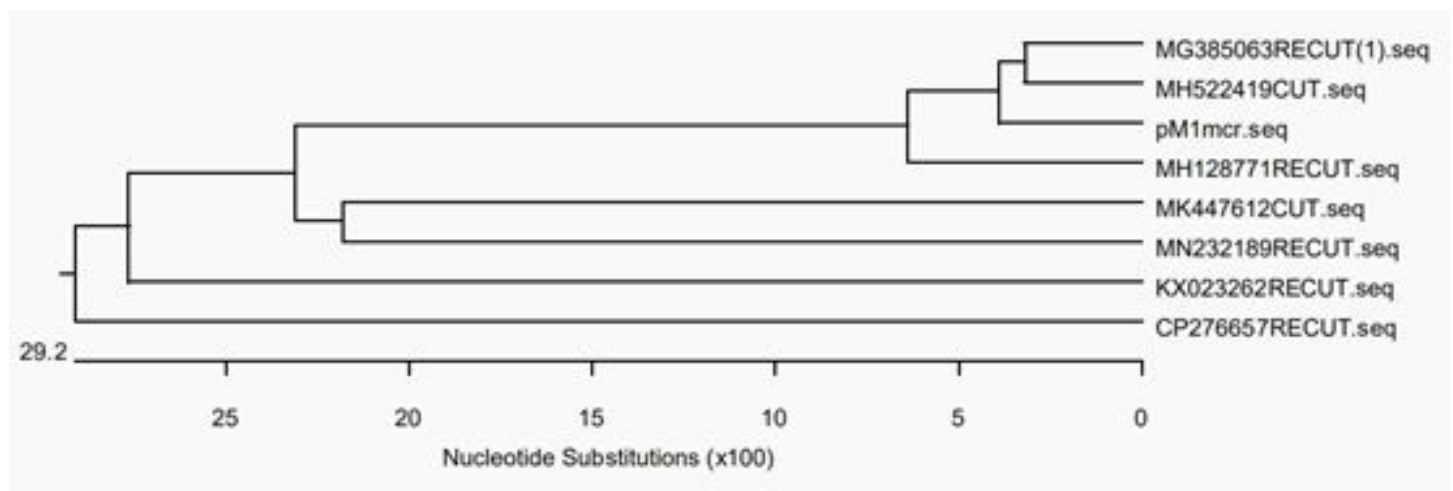

Figure 1 
Phylogenetic tree of pM1mcr and similar plasmids.

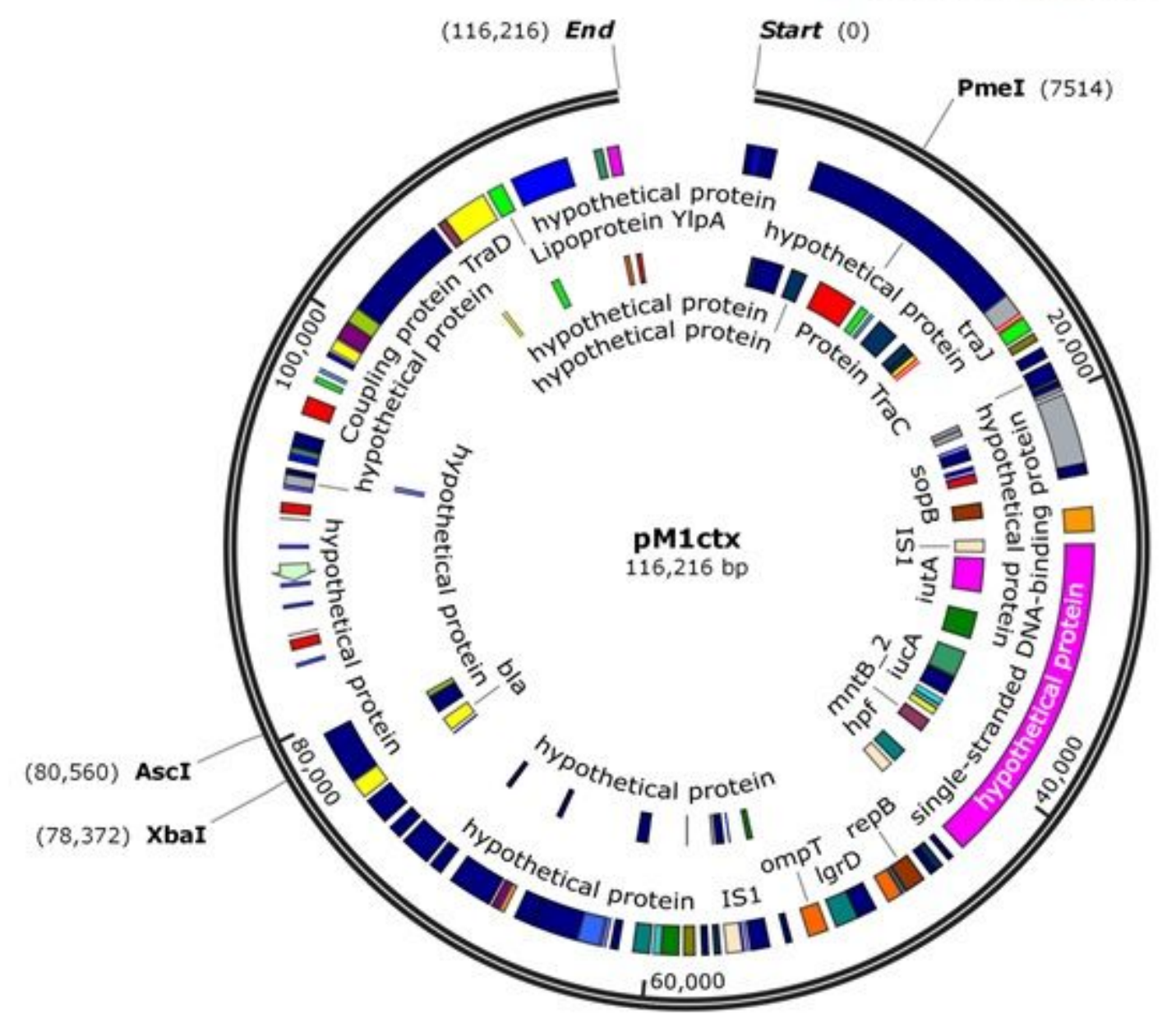

Figure 2

Structure of pM1ctx from Escherichia coli Mink 1. 